\title{
AN ISOPERIMETRIC INEQUALITY AND THE GEOMETRIC SOBOLEV EMBEDDING FOR VECTOR FIELDS
}

\author{
Luca Capogna, Donatella Danielli, and Nicola Garofalo
}

\section{Introduction}

The classical embedding theorem of Sobolev $W^{1, p}\left(\mathbb{R}^{n}\right) \hookrightarrow L^{q}\left(\mathbb{R}^{n}\right)$ was originally proved in the case $1<p<n$, with $q=\frac{n p}{n-p}$. It was only in the late fifties that by means of an elegant integral inequality Gagliardo and Nirenberg were independently able to obtain the limiting case $p=1$ and prove that: $(*) W^{1,1}\left(\mathbb{R}^{n}\right) \hookrightarrow L^{\frac{n}{n-1}}\left(\mathbb{R}^{n}\right)$ (see, e.g., $[\mathrm{S}]$ ). Meanwhile, in his fundamental work [DG] De Giorgi introduced the notion of total variation of an $L^{1}$ distribution and laid down his theory of generalized perimeters. Soon after, Fleming and Rishel [FR] gave a beautiful geometric new proof of $(*)$ based on Federer's co-area formula and the celebrated isoperimetric inequality: $(* *) P(E) \geq c_{n}|E|^{\frac{n-1}{n}}$, where $P(E)$ denotes the perimeter according to De Giorgi and $c_{n}=n \Gamma\left(\frac{1}{2}\right) \Gamma\left(\frac{n}{2}+1\right)^{-\frac{1}{n}}$. It turns out that, in fact, $(*)$ is equivalent to $(* *)$, see e.g. [T].

The purpose of this note is to announce an optimal embedding theorem similar to $(*)$ for the Sobolev spaces associated to some general families of vector fields satisfying certain geometric conditions. A priori, we do not need to require $C^{\infty}$ smoothness of the vector fields. To keep some unity of presentation, however, we have confined our discussion to the case of $C^{\infty}$ vector fields satisfying Hörmander's condition on the Lie algebra $[\mathrm{H}]$. For instance, operators of Baouendi-Grushin type

$$
\mathcal{L}_{\alpha}=\Delta_{z}+|z|^{2 \alpha} \Delta_{t}, \quad z \in \mathbb{R}^{n}, \quad t \in \mathbb{R}^{m}, \quad \alpha>0,
$$

are a typical example of non-Hörmander type operators to which our results apply.

Received November 20, 1993.

N. Garofalo was supported by NSF Grant DMS 9104023. 
In our general setting, the idea of Gagliardo and Nirenberg does not apply. Instead, we have proved a notable isoperimetric inequality (Theorem 5) whose dimensional optimality is easily tested in the case of nilpotent homogeneous Lie groups. We mention that, in the special context of the Heisenberg group $\mathbb{H}^{1} \simeq \mathbb{C} \times \mathbb{R}$, Pansu $[\mathrm{P}]$ had proved an isoperimetric inequality. His method, however, is different from ours. Our approach departs slightly from the classical one in that we infer the isoperimetric inequality from a weak version of the Sobolev embedding (Theorem 2). Then, we use it to prove the strong embedding (Theorem 1). The latter, in turn, implies the isoperimetric inequality, thus closing the circle. As it is well known the case $p=1$ of the Sobolev embedding is the strongest result of its category, in the sense that, from it, the case $p>1$ with optimal exponents can be trivially obtained.

A distinctive aspect of our approach is that the geometric Sobolev embedding (Theorem 1) is deduced from two rather general facts: (I) The doubling condition (2.1) for the balls in the Carnot-Carathédory metric associated to the vector fields; (II) The possibility of representing compactly supported functions in a ball, in terms of a metric fractional integral involving the (degenerate) gradient associated to the fields, see (2.3). We mention that interpolation is never used in the course of the proofs.

\section{Statements of the Results}

Let $X_{1}, \ldots, X_{m}$ be $C^{\infty}$ vector fields in $\mathbb{R}^{n}$ satisfying Hörmander's condition for hypoellipticity $[\mathrm{H}]: \operatorname{rank} \operatorname{Lie}\left[X_{1}, \ldots, X_{m}\right]=n$ at every point. The Carnot-Carathéodory distance associated to $\left(X_{1}, \ldots, X_{m}\right)$ is defined by $d(x, y)=\inf \left\{T>0 \mid\right.$ There exists a sub-unitary curve $\gamma:[0, T] \rightarrow \mathbb{R}^{n}$ such that $\gamma(0)=x, \gamma(T)=y\}$. We recall that a piecewise $C^{1}$ curve $\gamma:[0, T] \rightarrow \mathbb{R}^{n}$ is called sub-unitary if for every $\xi \in \mathbb{R}^{n}$ and $t \in(0, T)$ for which $\gamma^{\prime}(t)$ is defined one has: $\left\langle\gamma^{\prime}(t), \xi\right\rangle^{2} \leq \sum_{j=1}^{m}\left\langle X_{j}(\gamma(t)), \xi\right\rangle^{2}$. Denote by $B(x, R)=\left\{y \in \mathbb{R}^{n} \mid d(x, y)<R\right\}$. A fundamental consequence of the works $[\mathrm{FP}]$ and [NSW] is the following: Given a bounded set $U \subset \mathbb{R}^{n}$ there exist $C_{1}>0$ and $R_{0}>0$ such that for any $x \in U, 0<R \leq R_{0}$

$$
|B(x, 2 R)| \leq C_{1}|B(x, R)| .
$$

An immediate corollary of (2.1) is the existence of a number $Q>0$ such that: For any $x \in U, 0<R \leq R_{0}$ and $0<t<1$

$$
|B(x, R)| \leq C_{1} t^{-Q}|B(x, t R)| .
$$

We note that $Q=\frac{\log C_{1}}{\log 2}$, with $C_{1}$ as in (2.1), so that in the case of a nilpotent, homogeneous Lie group, the number $Q$ in (2.2) is constant 
throughout the group and is, in fact, the homogeneous dimension of the group, see [FS]. For this reason we call the number $Q$ in (2.2) the local homogeneous dimension (of the vector fields $X_{1}, \ldots, X_{m}$ ) relative to $U$. In what follows for a function $u$ denote by $X u=\left(X_{1} u, \ldots, X_{m} u\right)$ its so-called subelliptic gradient. For $1 \leq p<\infty$ consider the functional

$$
J_{p, \Omega}(u)=\int_{\Omega}|X u|^{p} d x=\int_{\Omega}\left[\sum_{j=1}^{m}\left(X_{j} u\right)^{2}\right]^{\frac{p}{2}} d x,
$$

where $\Omega \subset \mathbb{R}^{n}$ is an open set. We define $\stackrel{\circ}{S}^{1, p}(\Omega)$ to be the completion of $C_{0}^{1}(\Omega)$ in the norm $\|u\|_{S^{1, p}(\Omega)}=\left[J_{p, \Omega}(u)+\|u\|_{L^{p}(\Omega)}^{p}\right]^{1 / p}$.

The main result in this note is the following:

Theorem 1. Let $U \subset \mathbb{R}^{n}$ be a bounded set and $Q$ be the local homogeneous dimension relative to $U$. There exist $C_{2}>0$ and $R_{0}>0$ such that for any $x \in U, B_{R}=B(x, R)$, with $0<R \leq R_{0}$, and every $u \in \stackrel{\circ}{S}^{1,1}\left(B_{R}\right)$ one has

$$
\left(\frac{1}{\left|B_{R}\right|} \int_{B_{R}}|u|^{k} d x\right)^{\frac{1}{k}} \leq C_{2} R\left(\frac{1}{\left|B_{R}\right|} \int_{B_{R}}|X u| d x\right),
$$

where $1 \leq k \leq \frac{Q}{Q-1}$.

Remark. The exponent $\frac{Q}{Q-1}$ in the left-hand side of the above inequality is optimal. This can be easily tested in the case of nilpotent homogeneous Lie groups.

Our strategy to prove Theorem 1 is based on the following weak form of the latter.

Theorem 2. Let $U \in \mathbb{R}^{n}$ be a bounded set and $Q$ be the local homogeneous dimension relative to it. There exist $C>0$ and $R_{0}>0$ such that for any $x \in U, B_{R}=B(x, R)$, with $0<R \leq R_{0}$, and every Lipschitz function $u$ compactly supported in $B_{R}$ one has

$$
\left|\left\{x \in B_{R}|| u(x) \mid>\lambda\right\}\right| \leq \frac{C}{\lambda^{q}} R^{q}\left|B_{R}\right|^{1-q}\|X u\|_{L^{1}\left(B_{R}\right)}^{q}
$$

for any $\lambda>0$. Here $q=\frac{Q}{Q-1}$.

The proof of Theorem 2 is only based on the following two ingredients:

(I) The size estimate $(2.1)$ of the metric balls $B(x, R)$.

(II) A notable representation formula for any Lipschitz function $u$ compactly supported in $B(x, R)$, see [D], [CDG]. This formula reads

$$
|u(y)| \leq C I_{1}(|X u|)(y), \quad y \in B(x, R) .
$$


Here, for every $0<\alpha<Q, I_{\alpha}$ is the metric fractional integration operator

$$
I_{\alpha} f(y)=\int_{B(x, R)}|f(\xi)| \frac{d(y, \xi)^{\alpha}}{|B(y, d(y, \xi))|} d \xi, \quad y \in B(x, R) .
$$

At this point we introduce a suitable generalization of the notion of total variation due to De Giorgi [DG], see also [FR].

Definition 3. Let $u \in L^{1}(\Omega)$. We define the $X$-variation of $u$ in $\Omega$ as

$$
\operatorname{Var}_{X}(u ; \Omega)=\sup _{\varphi \in \mathcal{F}} \int_{\Omega} u \sum_{j=1}^{m} X_{j}^{*} \varphi_{j} d x,
$$

where $X_{j}^{*}$ denotes the formal adjoint of the vector field $X_{j}$ and $\mathcal{F}$ denotes the class of all functions $\varphi=\left(\varphi_{1}, \ldots, \varphi_{m}\right) \in C_{0}^{1}(\Omega)^{m}$ such that $\|\varphi\|_{\infty}=$ $\sup _{x \in \Omega}\left(\sum_{j=1}^{m}\left|\varphi_{j}(x)\right|^{2}\right)^{\frac{1}{2}} \leq 1$.

Definition 4. Let $E$ be a bounded measurable set in $\mathbb{R}^{n}$. The $X$-perimeter of $E$ with respect to $\Omega$ is defined as $\operatorname{Var}_{X}\left(\chi_{E} ; \Omega\right)$, where $\chi_{E}$ is the characteristic function of $E$.

We will denote by $P_{X}(E ; \Omega)$ the $X$-perimeter of $E$ with respect to $\Omega$.

Remark. Suppose that $E$ is a bounded open set in $\mathbb{R}^{n}$ with $C^{1}$ boundary. Then one has

$$
P_{X}(E ; \Omega)=\int_{\partial E \cap \Omega}\left[\sum_{j=1}^{m}\left\langle X_{j}, \eta\right\rangle^{2}\right]^{\frac{1}{2}} d H_{n-1},
$$

where $\eta$ indicates the outward unit normal to $\partial E$ and $d H_{n-1}$ the $(n-1)$ dimensional Hausdorff measure in $\mathbb{R}^{n}$.

Applying Theorem 2 and the previous remark we obtain the following remarkable

Theorem 5. (Sub-elliptic isoperimetric inequality.) Let $U \subset \mathbb{R}^{n}$ be a bounded set and $Q$ be the local homogeneous dimension relative to $U$. There exist constants $C>0$ and $R_{0}>0$ such that for any $x \in U$ and $B_{R}=$ $B(x, R)$, with $0<R \leq R_{0}$, and for every $C^{1}$ open subset $E \subset \subset B_{R}$, one has

$$
|E|^{\frac{Q-1}{Q}} \leq C \frac{R}{\left|B_{R}\right|^{1 / Q}} P_{X}\left(E ; B_{R}\right) .
$$

It is worth observing that, because of $(2.2)$, the product $R\left|B_{R}\right|^{-\frac{1}{Q}}$ is bounded uniformly by a constant which is independent of $x \in U$ and $R \leq$ $R_{0}$. 
With Theorem 5 in hand we can prove Theorem 1 following a classical argument (see $[\mathrm{FR}]$ ), after having observed that

$$
\int_{B_{R}}|X u| d x=\int_{0}^{\infty} P_{X}\left(E_{t} ; B_{R}\right) d t
$$

with $E_{t}=\left\{x \in B_{R} \mid u(x)>t\right\}, u \in C_{0}^{1}\left(B_{R}\right), u \geq 0$.

We close by remarking that the results of this note hold for any family of vector fields on $\mathbb{R}^{n}$ (or more generally on a connected manifold $M^{n}$ ) for which a natural distance can be defined which generates a structure of space of homogeneous type, see $[\mathrm{CW}]$. And for which, furthermore, a representation formula such as (2.3) above holds. Now, (2.1) and (2.3) can be proved for wide classes of families of vector fields under minimal smoothness assumptions on the coefficients.

\section{References}

[CDG] L. Capogna, D. Danielli, and N. Garofalo, An embedding theorem and the Harnack inequality for nonlinear subelliptic equations, Comm. P. D. E. 18 (1993), 1765-1794.

[CW] R. R. Coifman and G. Weiss, Analyse harmonique non-commutative sur certain spaces homogènes. Etudes de certaines intégrals singuliéres, Lecture Notes in Mathematics, vol. 242, Springer-Verlag, Berlin, New York, 1971.

[D] D. Danielli, Formules de représentation et théorèmes d'inclusion pour des opérateurs sous-elliptiques, C. R. de l'Acad. des Sc., Paris, 314, Serie I (1992), 987-990, 987-990.

[DG] E. De Giorgi, Su una teoria generale della misura $(r-1)$ dimensionale in uno spazio ad $r$ dimensioni, Ann. Mat. Pura Appl. 36 (1954), 191-213.

[FE] H. Federer, Geometric Measure Theory, Springer-Verlag, Berlin, Heidelberg, New York, 1969.

[FP] C. Fefferman and D. H. Phong, Subelliptic eigenvalue problems, Proceedings of the Conference in Harmonic Analysis in Honor of A. Zygmund, Wadsworth Math. Ser., Wadsworth, Belmont, CA, 1981, pp. 590-606.

[FR] W. H. Fleming and R. Rishel, An integral formula for total gradient variation, Arch. Math. 11 (1960), 218-222.

[FS] G. B. Folland and E. M. Stein, Hardy Spaces on Homogeneous Groups, Math. Notes, Princeton Univ. Press, 1982.

[H] L. Hörmander, Hypoelliptic second order differential equations, Acta Math. 119 (1967), 147-171.

[NSW] A. Nagel, E. M. Stein, and S. Wainger, Balls and metrics defined by vector fields I: Basic properties, Acta Math. 155 (1985), 103-147.

[P] Pansu P., Une inégalité isopérimétrique sur le groupe de Heisenberg, C. R. de l'Acad. des Sc., Paris 295, Serie I (1982), 127-130.

[SC] A. Sanchez Calle, Fundamental solutions and geometry of sum of squares of vector fields, Inv. Math. 78 (1984), 143-160.

[S] E. M. Stein, Singular Integrals and Differentiability Properties of Functions, Princeton Univ. Press, Princeton, 1970. 
[T] G. Talenti, Best constants in Sobolev inequality, Ann. Mat. Pura Appl. 110 (1976), 353-372.

Department of M athematics, Purdue Univeritit, West Lafayette, In 47907

E-mail address: capogna@math.purdue.edu,dxd@math.purdue.edu, garofalo@math.purdue.edu 